\title{
NONLINEAR FILTERING AND LARGE DEVIATIONS *
}

\author{
M. R. James J. S. Baras \\ Systems Research Center \\ University of Maryland \\ College Park MD 20742
}

\begin{abstract}
We consider the nonlinear filtering problem $d x=f(x) d t+$ $\sqrt{\epsilon} d w, d y=h(x) d t+\sqrt{\epsilon} d v$, and obtain $\lim _{\epsilon \rightarrow 0} \epsilon \log q^{\epsilon}(x, t)=$ $-W(x, t)$ for unnormalised conditional densities $q^{\epsilon}(x, t)$ using PDE methods. Here, $W(x, t)$ is the value function for a deterministic optimal control problem arising in Mortensen's deterministic estimation, and is the unique viscosity solution of a Hamilton-Jacobi-Bellman equation.
\end{abstract}

\section{Introduction}

An important problem in system theory is the construction of observers for nonlinear control systems. Baras, Bensoussan and James [1] have studied a method for constructing an observer as a limit of nonlinear filters for a family of associated filtering problems (2), parameterised by $\epsilon>0$. It is of interest then to study the asymptotic behaviour of the corresponding unnormalised conditional densities $q^{\epsilon}(x, t)$ as $\epsilon \rightarrow 0$, via the Zakai equation (3). We obtain the asymptotic formula

$$
q^{\epsilon}(x, t)=e^{-\frac{1}{\varepsilon}(W(x, t)+o(1))},
$$

as $\epsilon \rightarrow 0$, where $W(x, t)$ is the value function corresponding to a deterministic optimal control problem, namely that arising in deterministic estimation.

Our method is inspired by the work of Fleming and Mitter [4], and Evans and Ishii [3]. A logarithmic transformation is applied to the robust form of the Zakai equation, yielding a Hamilton-Jacobi equation in the limit. A related HamiltonJacobi equation is interpreted as the Bellman equation for the optimal control problem arising in deterministic estimation, of which $W(x, t)$ is the unique viscosity solution. In particular, $W(x, t)$ is not assumed to be smooth.

This problem has been studied by Hijab [5] using different methods. Hijab also obtained a large deviation principle for conditional measures on $C\left([0, T] ; \mathbb{R}^{n}\right)$. An extension of his result is presented in James and Baras [6], which includes complete proofs of the results discussed in the present paper.

\section{Problem Formulation}

We consider a family of diffusion processes in $\mathbb{R}^{n}$ with real valued observations:

$$
\begin{aligned}
& d x^{\epsilon}(t)=f\left(x^{\epsilon}(t)\right) d t+\sqrt{\epsilon} d w(t), \quad x^{\epsilon}(0)=x_{0}^{\epsilon}, \\
& d y^{\epsilon}(t)=h\left(x^{\epsilon}(t)\right) d t+\sqrt{\epsilon} d v(t), \quad y^{\epsilon}(0)=0 .
\end{aligned}
$$

Here $w, v$ are independent Wiener processes independent of the initial conditions $x_{0}^{\epsilon}$, which have (unnormalised) densities $q_{0}^{\epsilon}(x)=C_{\epsilon} e^{-\frac{1}{\epsilon} S_{0}(x)}$ where $\lim _{\epsilon \rightarrow 0} \epsilon \log C_{\epsilon}=0$ and $S_{0} \geq 0$ is smooth and bounded. As $\epsilon \rightarrow 0$ the trajectories of (2) converge in probability to the trajectory of a corresponding deterministic system. We assume throughout the following: $f, h$ are bounded $C^{\infty}$ functions with bounded derivatives of orders 1 and 2 .

The Zakai equation for an unnormalised conditional density $q^{\epsilon}(x, t)$ is

$$
\begin{aligned}
d q^{\epsilon}(x, t) & =A_{\epsilon}^{*} q^{\epsilon}(x, t)+\frac{1}{\epsilon} h(x) q^{\epsilon}(x, t) d y^{\epsilon}(t), \\
q^{\epsilon}(x, 0) & =q_{0}^{\epsilon}(x),
\end{aligned}
$$

where $A_{\epsilon}^{*}$ is the formal adjoint of the diffusion operator. Defining

$$
p^{\epsilon}(x, t)=\exp \left(-\frac{1}{\epsilon} y^{\epsilon}(t) h(x)\right) q^{\epsilon}(x, t),
$$

the robust form of the Zakai equation is

$$
\begin{gathered}
\frac{\partial}{\partial t} p^{\epsilon}(x, t)-\frac{\epsilon}{2} \Delta p^{\epsilon}(x, t)+D p^{\epsilon}(x, t) g^{\epsilon}(x, t)+\frac{1}{\epsilon} V^{\epsilon}(x, t) p^{\epsilon}(x, 0)=0, \\
p^{\epsilon}(x, t)=q_{0}^{\epsilon}(x) .
\end{gathered}
$$

Note that (5) is a linear parabolic PDE and the coefficient $V^{\epsilon}$ depends on the observation path $t \mapsto y(t)$. We shall omit the $\epsilon$-dependence of $y$, and view (5) as a functional of the observation path $y \in \Omega_{0}=C\left([0, T], \mathbb{R}^{n} ; y(0)=0\right)$. This transformation provides a convenient choice of a version of the conditional density, and under our assumptions we can recover the unnormalised density $q^{\epsilon}(x, t)$ from the solution of (5).

Following Fleming and Mitter [4], who considered filtering problems with $\epsilon=1$, we apply the logarithmic transformation

$$
S^{\epsilon}(x, t)=-\epsilon \log p^{\epsilon}(x, t) .
$$

Then $S^{\epsilon}(x, t)$ satisfies

$$
\begin{gathered}
\frac{\partial}{\partial t} S^{\epsilon}(x, t)-\frac{\epsilon}{2} \Delta S^{\epsilon}(x, t)+H^{\epsilon}\left(x, t, D S^{\epsilon}(x, t)\right)=0, \\
S^{\epsilon}(x, 0)=S_{0}(x),
\end{gathered}
$$

where

$$
H^{\epsilon}(x, t, \lambda)=\lambda g^{\epsilon}(x, t)+\frac{1}{2}|\lambda|^{2}-V^{\epsilon}(x, t) .
$$

Equation (7) is a nonlinear parabolic PDE. Formally letting $\epsilon \rightarrow 0$ we obtain a Hamilton-Jacobi equation

$$
\begin{gathered}
\frac{\partial}{\partial t} S(x, t)+H(x, t, D S(x, t))=0, \\
S(x, 0)=S_{0}(x),
\end{gathered}
$$


where

$$
H(x, t, \lambda)=\lambda g_{0}(x, t)+\frac{1}{2}|\lambda|^{2}-V(x, t),
$$

Note that $g^{\epsilon} \rightarrow g_{0}, V^{\epsilon} \rightarrow V$, and $H^{\epsilon} \rightarrow H$ uniformly on compact subsets. We shall interpret solutions of (9) in the viscosity sense. If we define

$$
W(x, t)=S(x, t)-y(t) h(x), y \in \Omega_{0},
$$

then, for $y \in \Omega_{0} \cap C^{1}, W(x, t)$ satisfies a Hamilton-Jacobi equation, which is presented as the Bellman equation for the deterministic estimation control problem below.

\section{Deterministic Estimation}

We begin by reviewing Mortensen's method [5] of deterministic minimum energy estimation. Given an observation record $y_{t}=$ $\{y(s), 0 \leq s \leq t\}, 0 \leq t \leq T$, of the deterministic system

$$
\begin{aligned}
& \dot{x}=f(x)+u, x(0)=x_{0}, \\
& \dot{y}=h(x)+v, y(0)=0,
\end{aligned}
$$

we wish to estimate the state at time $t$, the initial condition $x_{0}$ being unknown. Define

$$
J_{t}\left(x_{0}, u\right)=S_{0}\left(x_{0}\right)+\int_{0}^{t} L(x(s), u(s), s) d s,
$$

where

$$
L(x, u, s)=\frac{1}{2}|u|^{2}+\frac{1}{2} h(x)^{2}-\dot{y}(s) h(x) .
$$

We now minimise $J_{t}$ over pairs $\left(x_{0}, u\right)$. The deterministic or minimum energy estimate $\hat{x}(t)$ given $y_{t}$ is defined to be the endpoint of the optimal trajectory $s \mapsto x^{\prime \prime}(s), 0 \leq s \leq t$, corresponding to a minimum energy pair $\left(x_{0}^{*}, u^{*}\right): \hat{x}(t)=x^{*}(t)$.

We use dynamic programming to study this problem. Define a value function

$$
W(x, t)=\inf _{\left(x_{0}, u\right)}\left\{J_{t}\left(x_{0}, u\right): x(0)=x_{0}, x(t)=x\right\} .
$$

By using standard methods, we see that $W(x, t)$ is continuous and formally satisfies the Bellman equation

$$
\begin{gathered}
\frac{\partial}{\partial t} W(x, t)+\tilde{H}(x, t, D W(x, t))=0, \\
W(x, 0)=S_{0}(x),
\end{gathered}
$$

where

$$
\tilde{H}(x, t, \lambda)=\max _{u \in U}\{\lambda(f(x)+u)-L(x, u, t)\} .
$$

To obtain $\hat{x}(t)$, one minimises $W(x, t)$ over $x$. In fact, using the definition of viscosity solutions in Crandall, Evans and Lions $[2]$, we can prove:

Theorem The value function $W(x, t)$ defined by $(15)$ is the unique viscosity solution of the Hamilton-Jacobi-Bellman equation (16). In addition, the function $S(x, t)$ defined by $(6)$ is the unique viscosity solution of the Hamilton-Jacobi equation (9).

\section{Some Estimates}

Let $S^{\epsilon}(x, t)$ be the solution of (7). The following estimates are used to prove that $S^{\epsilon} \rightarrow S$.
Theorem For every compact subset $Q \subset \mathbb{R}^{n} \times[0, T]$, there exists $\epsilon_{0}>0$ and $K>0$ such that for $0<\epsilon<\epsilon_{0}$ we have

$$
\begin{aligned}
&\left|S^{\epsilon}(x, t)\right| \leq K, \text { for all }(x, t) \in Q, \\
&\left|D S^{\epsilon}(x, t)\right| \leq K, \text { for all }(x, t) \in Q .
\end{aligned}
$$

To prove (18), we use a comparison theorem which depends on the maximum principle for linear parabolic PDE. The gradient estimate (19) uses a variant of the techniques presented in Evans and Ishii [3], as suggested to us by L. C. Evans.

\section{Main Result}

We are now in a position to state and prove our main result.

Theorem Under the above assumptions, we have

$$
\lim _{\epsilon \rightarrow 0} \epsilon \log q^{\epsilon}(x, t)=-W(x, t)
$$

uniformly on compact subsets of $\mathbb{R}^{n} \times[0, T]$, where $W(x, t)$ is defined by (11).

Proof: From the above estimates and the Arzela-Ascoli theorem, there is a subsequence $\epsilon_{k} \rightarrow 0$ such that $S^{\epsilon_{k}}$ converges uniformly on compact subsets to a continuous function $\tilde{S}$. By the "vanishing viscosity" theorem [3], $\tilde{S}$ is a viscosity solution of (9). By uniqueness, $\tilde{S}=S$. In fact, $S^{\epsilon} \rightarrow S$ as $\epsilon \rightarrow 0$.

From this we have

$$
\lim _{\varepsilon \rightarrow 0} \epsilon \log q^{\epsilon}(x, t)=-(S(x, t)-y(t) h(x))
$$

uniformly on compact subsets, for $y \in \Omega_{0}$. Using the definition (11) of $W(x, t)$ completes the proof.

\section{References}

(1] J. S. Baras, A. Bensoussan and M. R. James, Dynamic Observers as Asymptotic Limits of Recursive Filters: Special Cases, SRC-TR-86-79, Systems Research Center, Univ. of MD, Dec. 1986. To appear, SIAM J. Applied Math.

[2] M. G. Crandall, L. C. Evans and P. L. Lions, Some Properties of Viscosity Solutions of Hamilton-Jacobi Equations, Trans. AMS, 282, No.2, April 1984, pp487-502.

[3] L. C. Evans and H. Ishii, A PDE approach to some asymp. totic problems concerning random differential equations with small noise intensities, Ann. Inst. Henri PoincaréAnalyse non linéire, 2, No.1, 1985, pp1-20.

[4] W. H. Fleming and S. K. Mitter, Optimal Control and Nonlinear Filtering for Nondegenerate Diffusion Processes, Stochastics, 8, 1982, pp63-77.

[5] O. Hijab, Minimum Energy Estimation, PhD Dissertation, University of California, Berkely, December, 1980.

[6] M. R. James and J. S. Baras, Nonlinear Filtering and Large Deviations: A PDE-Control Theoretic Approach, SRCTR-87-27, Systems Research Center, Univ. of MD, Feb. 1987.

* Research partially supported by NSF grant CDR-85-00108, and AFOSR contract AFOSR-870073. 